\title{
Evaluation de la toxicité d'un aphrodisiaque ivoirien d'origine naturelle (aphro) chez le rat
}

\author{
Moussa GBOGBO ${ }^{1,2 *}$, Rufin Yao KOUADIO ${ }^{1}$, Félicité Tano-bla $\mathrm{ABOLI}^{1}$, Mama $\mathrm{KONE}^{2}$, \\ Elisée Kouassi KPOROU ${ }^{1}$ et Paul Angoué YAPO ${ }^{2}$ \\ ${ }^{1}$ Département de Biochimie et Microbiologie, Université Jean Lorougnon GUÉDÉ, BP 150 Daloa, \\ Côte d'Ivoire. \\ ${ }^{2}$ Laboratoire de Physiologie, Pharmacologie et Pharmacopée, Université Nangui ABROGOUA, 02 BP 801 \\ Abidjan 02, Côte d'Ivoire. \\ *Auteur correspondant ; E-mail : gbogbo_moussa@ujlg.edu.ci ; Tel : (+225)0707452930
}

\begin{tabular}{ccc}
\hline Received: 08-03-2021 & Accepted: 23-08-2021 & Published: 31-08-2021 \\
\hline
\end{tabular}

\section{RESUMÉ}

L'usage des plantes à des fins thérapeutiques est très ancien. De nos jours encore, elles sont utilisées par une frange partie de la population mondiale. La présente étude avait pour objectif d'étudier les effets toxiques de l'administration subaiguë d'un phytomédicament ivoirien Aphro vendu sur les marchés chez le rat Wistar albinos. Pour ce faire, le phytomédicament Aphro a été administré quotidiennement par voie orale à trois lots de rats, aux doses de 5,$75 ; 11,5$ et $23 \mathrm{mg} / \mathrm{kg}$ de poids corporel et cela pendant 28 jours. Le taux de croissance des rats, les paramètres hématologiques et biochimiques sanguins ont été étudiés. Les résultats de cette étude ont révélé qu'aux doses de 5,$75 ; 11,5$ et $23 \mathrm{mg} / \mathrm{kg}$ de poids corporel, le phytomédicament ne modifie pas de façon significative le taux d'accroissement de la masse corporelle et les paramètres hématologiques constitués du taux de globules rouges, de globules blancs, de plaquettes sanguines et le taux d'hémoglobine. Par ailleurs, des marqueurs hépatiques et lipidiques sériques des rats traités n'ont pas été perturbés par l'administration du phytomédicament comparativement au lot témoin. En revanche, la créatinine, l'un des marqueurs rénaux étudiés a connu une augmentation significative $(\mathrm{p}<0,05)$ chez les rats traités avec le phytomédicament aux doses de 11,5 et $23 \mathrm{mg} / \mathrm{kg}$ de poids corporel par rapport au lot témoin. Cette étude a permis de montrer que l'usage prolongé et à doses élevées de Aphro pourrait entraîner des effets nocifs sur les reins.

(C) 2021 International Formulae Group. All rights reserved.

Mots clés : Toxicité, Aphro, rat, hématologiques, biochimiques.

\section{Assessment of the toxicity of an ivoirian natural aphrodisiac (aphro) on rat}

\section{ABSTRACT}

The use of plants for therapeutic purposes is very old. Even today, they are used by a part of the world population. The objective of the present study was to evaluate the toxic effects of the subacute administration of an ivorian phytomedicine Aphro sold in the markets in the albino Wistar rat. To do this, the phytomedicine Aphro was administered orally daily to three batches of rats, at doses of $5.75 ; 11.5$ and $23 \mathrm{mg} / \mathrm{kg}$ of body weight for 28 days. Rats' growth rate, blood haematological 
and biochemical parameters were studied. The results of this study showed that at doses of $5.75 ; 11.5$ and $23 \mathrm{mg} / \mathrm{kg}$ of body weight, the phytomedicine does not significantly modify the rate of increase in body mass and the hematological parameters consisting of the level of red blood cells, white blood cells, blood platelets and the level hemoglobin. Furthermore, hepatic and serum lipid markers of the treated rats were not disturbed by the administration of the phytomedicine compared to the control group. In contrast, creatinine, one of the renal markers studied, experienced a significant increase ( $p$ $<0.05$ ) in rats treated with the phytomedicine at doses of $11.5 \mathrm{and} 23 \mathrm{mg} / \mathrm{kg}$ of body weight compared to the batch witness. This study showed that prolonged use and in high doses of Aphro could cause harmful effects on the kidneys.

(c) 2021 International Formulae Group. All rights reserved.

Keywords: Toxicity, rat, Aphro, hematology, biochemistry.

\section{INTRODUCTION}

L'utilisation des plantes médicinales dans le traitement des pathologies remonte à plusieurs millénaires. Elle connait aujourd'hui une croissance dans la plupart des pays du monde malgré le développement des médicaments de synthèse (Kroa et al., 2014). Dans tous les pays en voie de développements tels que la Côte d'Ivoire, l'utilisation de plantes médicinales est le moyen le plus utilisé pour résoudre les problèmes de santé publique surtout en milieu rural. Selon l'Organisation Mondiale de la Santé (OMS) plus de 80\% de la population africaine a recours à la médecine traditionnelle pour ses soins de santé primaire du fait de leur proximité et de leur accessibilité (OMS, 2002). La médecine traditionnelle bénéficie de l'image d'une médecine douce car les substances utilisées, notamment les plantes, sont considérées comme des produits naturels de traitement dénué de tout risque. Cependant, de récentes études ont montré que la phytothérapie n'est pas toujours sans risque. En effet, plusieurs rapports font état de par le monde, des effets secondaires graves enregistrés suite à l'utilisation des plantes médicinales (Kandé et al., 2018). Au nombre de ces effets secondaires tnt la néphrotoxicité due aux plantes telle que le genre Aristolochia, l'hépatotoxicité du kava kava ou de Teucrium chamedrys ou d'Hypericum perforatum (Stickel et al., 2005). L'utilisation de ces plantes est donc parfois à l'origine de problèmes de toxicité ou d'interaction pouvant causer des échecs thérapeutiques (Owens et al., 2014). Ces échecs thérapeutiques sont dus à des perturbations des métabolismes des différents organes et peuvent même provoquer la mort (Najem et al., 2018).

En réalité, la nature abrite toute une série de plantes toxiques, comprenant des plantes vénéneuses et hallucinogènes, capables de provoquer des symptômes graves (Najem et al., 2018). La sécurité et l'innocuité sont ainsi donc d'importants critères à prendre en compte avant l'administration des produits à base de plantes.

En Côte d'Ivoire, des études enquêtes ethnobotaniques ont permis de dresser une liste non exhaustive d'espèces végétales utilisées en médecine traditionnelle par les populations (Dro et al., 2013). Aussi, un nombre important de travaux portant sur les propriétés biologiques, pharmacologiques et phytochimiques des plantes issues de la médecine traditionnelle ivoirienne ont été réalisés. Les données fournies par ces études ont permis d'une part d'expliquer l'action thérapeutique et d'autre part de confirmer l'utilisation de ces différentes plantes en médicine traditionnelle. Cependant, le domaine de l'évaluation de la qualité et de l'innocuité des médicaments traditionnels vendus sur le marché ivoirien reste peu exploré. La valorisation scientifique de la médecine traditionnelle doit conduire notamment à la mise au point de médicaments à base de plantes dont la sécurité, l'efficacité et la qualité sont certifiés (OMS, 2004). C'est dans ce cadre que s'est inscrit le présent travail qui avait pour but d'initier une étude toxicologique d'un médicament traditionnel à base de plantes 
vendu sur le marché ivoirien et utilisé comme aphrodisiaque.

\section{MATERIEL ET METHODES \\ Matériel \\ Animaux}

Des rats de l'espèce Ratus norvegicus de souche Wistar, d e sexe mâle et femelle (20 mâles et 20 femelles) âgés de huit semaines et pesant $135 \pm 15 \mathrm{~g}$ ont été utilisés pour les tests. Tous les animaux ont été soumis à une température de $25 \pm 2{ }^{\circ} \mathrm{C}$ et à une alternance de 12 heures de lumière et 12 heures d'obscurité. Les animaux avaient un accès libre à la nourriture et à l'eau. Le protocole expérimental et les procédures de manipulation animale ont été menés selon les bonnes pratiques de laboratoire (OCDE, 1998).

\section{Produit à administrer}

Le produit à tester est un médicament à base de plantes se présentant sous la forme liquide fourni par un tradithérapeute. La solution médicamenteuse (Aphro) a été filtrée, puis lyophilisée. La poudre obtenue, d'aspect brillant et de couleur marron, a été conservée puis utilisée pour les tests.

\section{Méthodes}

\section{Expérimentation animale}

L'évaluation de la toxicité subaiguë de Aphro chez les rats s'est effectuée par voie orale sur une durée de 28 jours. Au total, 4 lots de 10 rats comportant chacun 5 mâles et 5 femelles, mis séparément dans des cages, ont été constitués pour l'expérience (Irien'guessan et al., 2019). Le lyophilisat a été solubilisé dans de l'eau distillée et administré par gavage quotidiennement pendant les 4 semaines de l'étude aux doses de 5,4 - 11,5 et $23 \mathrm{mg} / \mathrm{kg}$ de poids corporel. Le lot témoin a reçu de l'eau distillée.

\section{Masse corporelle des rats}

Afin de suivre l'évolution corporelle des animaux le long de l'expérimentation, les animaux des différents groupes sont pesés avant l'expérience, au quatorzième et enfin au vingt-huitième jours de l'expérimentation. La masse corporelle est mesurée à l'aide d'une balance et le taux de croissance [TC (\%)] des rats par rapport au premier jour est exprimé en $\%$ et calculé selon la formule suivante :

TC $(\%)=\frac{(M j-M i) \times 100}{M i}$ où Mi est la masse initiale et $\mathrm{Mj}$ la masse du jour.

\section{Tests hématologiques}

Pour l'évaluation quantitative des trois lignées cellulaires hématologiques, trois prélèvements sanguins ont été effectués aux jours J0, J14 et J28. Le sang a été recueilli dans les tubes EDTA et a servi à la réalisation de l'hémogramme. Les globules rouges, les globules blancs, les plaquettes sanguines et l'hémoglobine ont été quantifiés.

\section{Tests biochimiques sériques}

Trois prélèvements sanguins, recueillis dans des tubes secs, ont été effectués aux jours J0, J14 et J28 pour réaliser des tests biochimiques sériques conformément aux méthodes usuelles de dosages. Ainsi, pour l'évaluation de quelques marqueurs biochimiques liés au foie, les paramètres suivants ont été dosés: Aspartate aminotransférase (ASAT) (Murray, 1984), Alanine aminotransférase (ALAT) (Murray, 1984), phosphatase alcaline (PAL) (Wenger et al., 1984 ; Rosalki et al., 1993) et Gammaglutamyltranspeptidase (rGT) (Tietz, 1999). Quelques paramètres associés aux reins tels que l'acide urique (Bernard, 1989), la créatinine (Murray, 1984), le calcium (Connerty et Briggs, 1966) et le magnésium (Farrell, 1984) ont été évalués. Le cholestérol total (Naito, 1984) et les triglycérides (Kaplan, 1984) ont été les paramètres lipidiques sériques évalués.

\section{Analyses statistiques}

Le logiciel GraphPad.Prism. V7.00 a été utilisé pour l'analyse statistique des données. Les données ont été analysées avec ANOVA un facteur. Les résultats sont présentés sous forme de moyenne \pm erreur standard sur la moyenne. La valeur de « $\mathrm{t} »$ nous donne le degré de significativité $« \mathrm{p} » l \mathrm{lu}$ sur la table de Student. La différence entre deux moyennes est considérée : significative : $\mathrm{p}<$ $0,05\left(^{*}\right)$; hautement significative $: p<0,01$ $(* *)$. 


\section{RESULTATS}

\section{Effet de Aphro sur le gain de masse corporelle des rats}

En comparaison avec la masse corporelle du premier jour de l'expérience, un gain de masse corporelle a été noté au bout des 28 jours chez les rats témoins ainsi que ceux qui ont été traités avec Aphro. Toutefois, les taux de croissance des rats traités à la dose 5,75 $\mathrm{mg} / \mathrm{kg}$ sont plus élevés par rapport aux autres lots mais non significatifs (Tableau 1).

\section{Effet de Aphro sur la variation des paramètres hématologiques}

Les résultats ont montré qu'aucune différence significative n'a été observée aux jours J0, J14 et J28 en ce qui concerne la variation du nombre de globules blancs, de globules rouges, des plaquettes sanguines et du taux d'hémoglobine entre les différents lots traités comparativement au lot témoin (Figure $1)$.

\section{Effet de Aphro sur quelques marqueurs biochimiques hépatiques}

L'administration quotidienne de Aphro aux doses de $5,75 \mathrm{mg} / \mathrm{kg}, 11,5$ et $23 \mathrm{mg} / \mathrm{kg}$ de poids corporel durant 28 jours n'a pas provoqué de perturbations significatives ( $\mathrm{p}>$ $0,05)$ sur les marqueurs hépatiques. Ainsi, l'aspartate aminotransférase (ASAT), l'alanine aminotransférase (ALAT), la phosphatase alcaline (PAL) et la gammaglutamyltranspeptidase (rGT) n'ont pas varié au cours de cette étude chez les rats traités par rapport aux rats témoins (Tableau 2).

\section{Effet de Aphro sur quelques marqueurs} biochimiques rénaux

L'administration quotidienne de Aphro durant 28 jours n'a pas perturbé les taux d'acide urique, de calcium et magnésium ( $\mathrm{p}>$ $0,05)$ chez les rats traités aux doses de 5,75 $\mathrm{mg} / \mathrm{kg}, 11,5$ et $23 \mathrm{mg} / \mathrm{kg}$ de poids corporel comparativement aux rats du lot témoin (Tableau 3). En revanche, une augmentation de la créatinémie a été observée au $28^{\text {ème jour }}$ respectivement dans les lots des rats traités avec le phytomédicament Aphro aux doses de $11,5 \mathrm{mg} / \mathrm{kg}(0,61 \pm 0,09 \mathrm{mg} / \mathrm{dl} ; \mathrm{p}<0,05)$ et 23 $\mathrm{mg} / \mathrm{kg}$ de poids corporel $(0,63 \pm 0,08 \mathrm{p}<0,05)$ par rapport au lot témoin $(0,43 \pm 0,08 \mathrm{mg} / \mathrm{dL})$ (Tableau 3).

\section{Effet de Aphro sur quelques paramètres lipidiques sériques}

Durant les 28 jours de l'essai, le cholestérol total et les triglycérides sériques n'ont pas été modifiés par l'administration du phytomédicament Aphro aux doses de 5,75 $\mathrm{mg} / \mathrm{kg}, 11,5$ et $23 \mathrm{mg} / \mathrm{kg}$ de poids corporel par rapport au lot témoin (Tableau 4).

Tableau 1: Évolution de la masse corporelle des rats traités avec Aphro comparativement au lot témoin

\begin{tabular}{|c|c|c|c|c|c|c|}
\hline Jours & Paramètres & $\begin{array}{l}\text { Lot } 1 \\
\text { (Témoin) }\end{array}$ & $\begin{array}{l}\text { Lot } 2 \\
(5,75 \mathrm{mg} / \mathrm{Kg})\end{array}$ & $\begin{array}{l}\text { Lot } 3 \\
(11,5 \mathrm{mg} / \mathrm{Kg})\end{array}$ & $\begin{array}{l}\text { Lot } 4 \\
(23 \mathrm{mg} / \mathrm{Kg})\end{array}$ & $\begin{array}{l}\text { Probabilité } \\
p\end{array}$ \\
\hline J0 & M. C (g) & $136,9 \pm 13,39$ & $121,6 \pm 14,23$ & $148,1 \pm 9,23$ & $\begin{array}{l}135,9 \pm 13,8 \\
9\end{array}$ & $p>0,05$ \\
\hline \multirow[t]{2}{*}{ J14 } & M. C (g) & $159 \pm 13,01$ & $145 \pm 10,22$ & $169,3 \pm 16,8$ & $\begin{array}{l}154,1 \pm 13,2 \\
3\end{array}$ & $P>0,05$ \\
\hline & T. C $(\%)$ & $+16,14$ & $+19,24$ & $+14,31$ & $+13,39$ & \\
\hline \multirow[t]{2}{*}{$\mathbf{J} 28$} & M. C (g) & $179,6 \pm 20,75$ & $170,8 \pm 7,54$ & $182,8 \pm 22,77$ & $\begin{array}{l}177,1 \pm 16,6 \\
7\end{array}$ & $P>0,05$ \\
\hline & T. C (\%) & $+12,95$ & $+17,79$ & $+7,97$ & $+14,92$ & \\
\hline
\end{tabular}

M. C : Masse Corporelle; T. C : Taux de Croissance; $\mathrm{p}=$ probabilité 
a

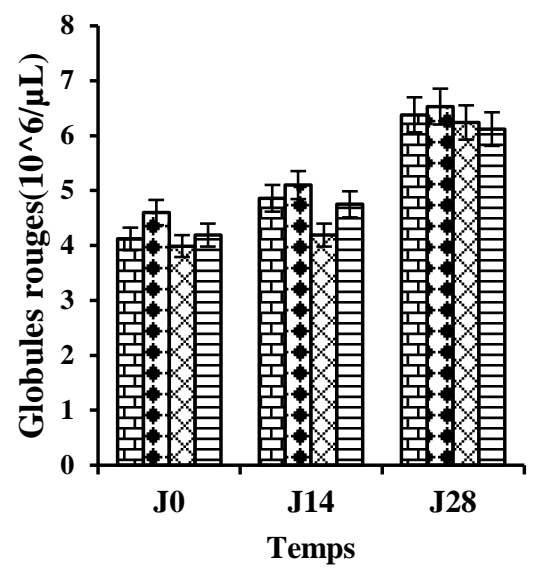

日Groupe-Témoin

- Groupe- $5,75 \mathrm{mg} / \mathrm{Kg}$ QGroupe-11,5 mg/Kg 口Groupe- $23 \mathrm{mg} / \mathrm{Kg}$
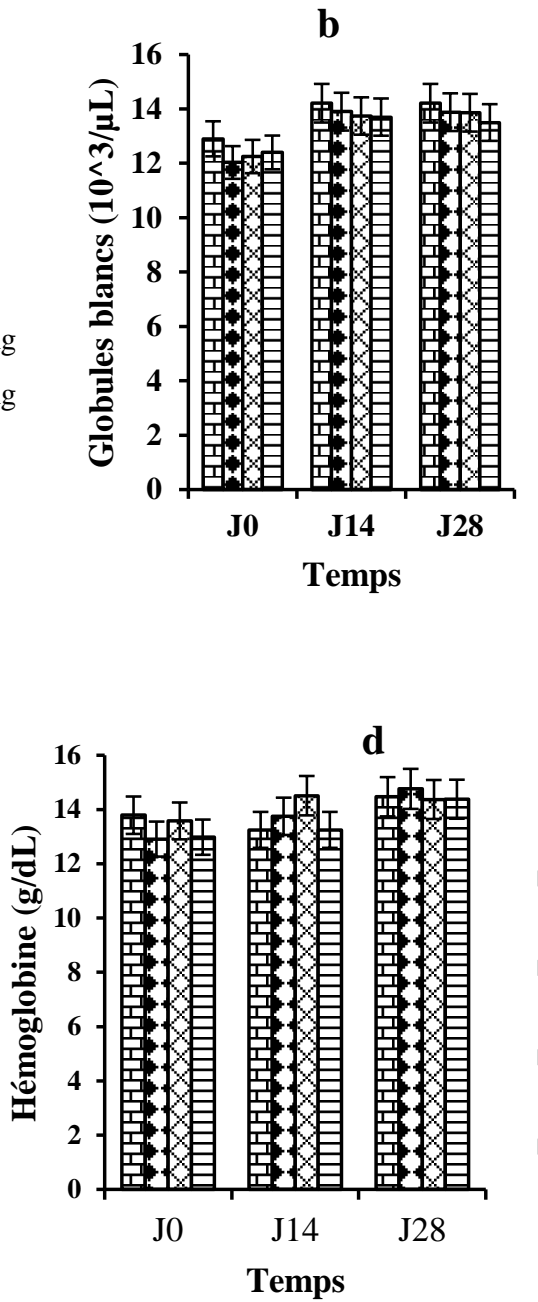

๑Groupe-Témoin

口Groupe-5,75 $\mathrm{mg} / \mathrm{Kg}$

-Groupe-11,5 $\mathrm{mg} / \mathrm{Kg}$

日Groupe-23 $\mathrm{mg} / \mathrm{Kg}$

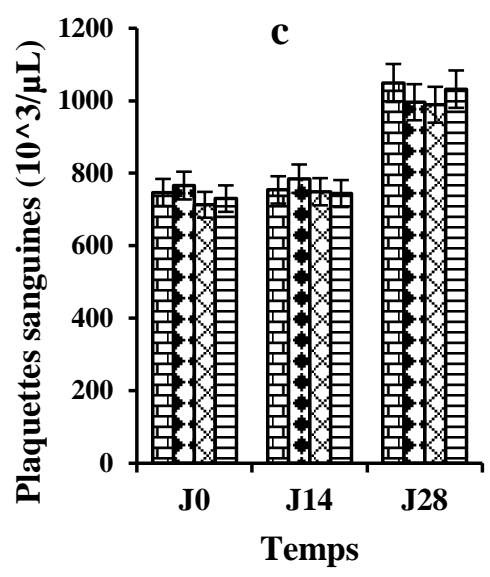

巴Groupe-Témoin

هGroupe-5,75 $\mathrm{mg} / \mathrm{Kg}$

ㅁ. Groupe-11,5 $\mathrm{mg} / \mathrm{Kg}$

口Groupe-23 mg/Kg
ĐGroupe-Témoin

๑roupe-5,75 $\mathrm{mg} / \mathrm{Kg}$

هGroupe-11,5 $\mathrm{mg} / \mathrm{Kg}$

口Groupe-23 mg/Kg

Figure 1 : Effet de l'administration de Aphro chez les rats sur: a = globules rouges, $\mathrm{b}=$ globules blancs, $\mathrm{c}=$ plaquettes sanguines, $\mathrm{d}=$ le taux d'hémoglobine, $\mathrm{J} 0=$ jour $0, \mathrm{~J} 14=$ jour $14, \mathrm{~J} 28=$ jour 28 , pas de différence significative $(p>0,05)$.

Tableau 2: Effet de Aphro sur quelques marqueurs biochimiques hépatiques chez les rats traités et les rats témoins.

\begin{tabular}{|c|c|c|c|c|}
\hline Paramètres & Lots & J0 & J14 & $\mathbf{J} 28$ \\
\hline \multirow{4}{*}{ ASAT (U/I) } & Lot 1 (Témoin) & $169,50 \pm 10$ & $168,60 \pm 14$ & $171,50 \pm 13$ \\
\hline & Lot $2(5,75 \mathrm{mg} / \mathrm{Kg})$ & $169,80 \pm 12$ & $170,80 \pm 10$ & $170,50 \pm 17$ \\
\hline & Lot $3(11,5 \mathrm{mg} / \mathrm{Kg})$ & $169,40 \pm 14$ & $170,60 \pm 12$ & $172,80 \pm 13$ \\
\hline & Lot $4(23 \mathrm{mg} / \mathrm{Kg})$ & $168,40 \pm 18$ & $172,40 \pm 14$ & $170,90 \pm 15$ \\
\hline \multirow{4}{*}{ ALAT (U/I) } & Lot 1 (Témoin) & $21,41 \pm 1,10$ & $20,10 \pm 1,30$ & $21,71 \pm 2,01$ \\
\hline & Lot $2(5,75 \mathrm{mg} / \mathrm{Kg})$ & $20,80 \pm 1,20$ & $21,70 \pm 1,40$ & $22,70 \pm 1,70$ \\
\hline & Lot $3(11,5 \mathrm{mg} / \mathrm{Kg})$ & $19,32 \pm 1,08$ & $20,10 \pm 1,20$ & $21,91 \pm 1,50$ \\
\hline & Lot $4(23 \mathrm{mg} / \mathrm{Kg})$ & $19,80 \pm 1,10$ & $21,70, \pm 1,30$ & $21,42 \pm 1,40$ \\
\hline \multirow[b]{2}{*}{ PAL (U/I) } & Lot 1 (Témoin) & $132,40 \pm 11$ & $138,40 \pm 10$ & $135,10 \pm 11$ \\
\hline & Lot $2(5,75 \mathrm{mg} / \mathrm{Kg})$ & $130,90 \pm 10$ & $134,20 \pm 13$ & $134,70 \pm 12$ \\
\hline
\end{tabular}




\begin{tabular}{l|llll} 
& Lot $3(11,5 \mathrm{mg} / \mathrm{Kg})$ & $130,70 \pm 12$ & $133,90 \pm 11$ & $135,70 \pm 10$ \\
& Lot $4(23 \mathrm{mg} / \mathrm{Kg})$ & $134,80 \pm 11$ & $133,70 \pm 10$ & $136,40 \pm 11$ \\
\hline \multirow{3}{*}{ rGT (U/I) } & Lot $1(\mathrm{Témoin})$ & $2,98 \pm 0,70$ & $2,63 \pm 0,74$ & $3,20 \pm 0,81$ \\
& Lot $2(5,75 \mathrm{mg} / \mathrm{Kg})$ & $3,01 \pm 0,88$ & $3,20 \pm 0,69$ & $3,10 \pm 0,76$ \\
& Lot $3(11,5 \mathrm{mg} / \mathrm{Kg})$ & $2,90 \pm 0,82$ & $2,97 \pm 0,65$ & $2,80 \pm 0,80$ \\
& Lot $4(23 \mathrm{mg} / \mathrm{Kg})$ & $2,80 \pm 0,80$ & $3,10 \pm 0,67$ & $3,00 \pm 0,69$ \\
\hline
\end{tabular}

ASAT : Aspartate Aminotransférase ; ALAT: Alanine Aminotransférase ; PAL : Phosphatase Alcaline ; rGT : Gammaglutamyltranspeptidase. $\mathrm{p}>0,05$ (pas de différence significative).

Tableau 3: Effet de Aphro sur quelques marqueurs biochimiques rénaux chez les rats traités et les rats témoins.

\begin{tabular}{l|llll}
\hline Paramètres & Lots & J0 & J14 & J28 \\
\hline \multirow{3}{*}{ CREAT $(\mathbf{m g} / \mathbf{d L})$} & Lot $1(\mathrm{Témoin})$ & $0,44 \pm 0,10$ & $0,47 \pm 0,10$ & $0,43 \pm 0,08$ \\
& Lot $2(5,75 \mathrm{mg} / \mathrm{Kg})$ & $0,41 \pm 0,09$ & $0,50 \pm 0,08$ & $0,58 \pm 0,07$ \\
& Lot $3(11,5 \mathrm{mg} / \mathrm{Kg})$ & $0,42 \pm 0,08$ & $0,54 \pm 0,07$ & $0,61 \pm 0,09^{*}$ \\
& Lot $4(23 \mathrm{mg} / \mathrm{Kg})$ & $0,41 \pm 0,09$ & $0,51 \pm 0,11$ & $0,63 \pm 0,08^{*}$ \\
\hline \multirow{3}{*}{ A. urique $(\mathbf{m g} / \mathbf{d L})$} & Lot $1(\mathrm{Témoin})$ & $4,90 \pm 0,88$ & $5,10 \pm 0,85$ & $4,80 \pm 0,97$ \\
& Lot $2(5,75 \mathrm{mg} / \mathrm{Kg})$ & $4,70 \pm 1,01$ & $5,19 \pm 1,02$ & $4,90 \pm 0,98$ \\
& Lot $3(11,5 \mathrm{mg} / \mathrm{Kg})$ & $4,95 \pm 1,08$ & $5,00 \pm 1,03$ & $5,10 \pm 0,87$ \\
& Lot $4(23 \mathrm{mg} / \mathrm{Kg})$ & $5,10 \pm 0,93$ & $5,01 \pm 0,90$ & $5,20 \pm 0,87$ \\
\hline \multirow{3}{*}{$\mathbf{C a}^{2+}(\mathbf{m g} / \mathbf{d L})$} & Lot $1(\mathrm{Témoin})$ & $6,30 \pm 1,03$ & $6,20 \pm 1,11$ & $6,60 \pm 1,00$ \\
& Lot $2(5,75 \mathrm{mg} / \mathrm{Kg})$ & $6,20 \pm 1,00$ & $6,30 \pm 0,98$ & $6,40 \pm 1,08$ \\
& Lot $3(11,5 \mathrm{mg} / \mathrm{Kg})$ & $6,40 \pm 0,99$ & $6,40 \pm 0,87$ & $6,50 \pm 1,12$ \\
& Lot $4(23 \mathrm{mg} / \mathrm{Kg})$ & $6,40 \pm 1,02$ & $6,30 \pm 0,79$ & $6,50 \pm 0,99$ \\
\hline $\mathbf{M g}^{\mathbf{2 +}}(\mathbf{g} / \mathbf{d L})$ & Lot $1(\mathrm{Témoin})$ & $3,10 \pm 0,56$ & $3,00 \pm 0,87$ & $3,40 \pm 0,67$ \\
& Lot $2(5,75 \mathrm{mg} / \mathrm{Kg})$ & $3,20 \pm 0,45$ & $3,10 \pm 0,69$ & $3,30 \pm 0,88$ \\
& Lot $3(11,5 \mathrm{mg} / \mathrm{Kg})$ & $3,20 \pm 0,75$ & $3,10 \pm 0,74$ & $3,30 \pm 0,39$ \\
& Lot $4(23 \mathrm{mg} / \mathrm{Kg})$ & $2,90 \pm 0,98$ & $3,20 \pm 0,84$ & $3,10 \pm 0,80$ \\
\hline
\end{tabular}

CREAT : Créatinine ; A. urique : Acide urique ; $\mathrm{Ca}^{2+}:$ Calcium ; $\mathrm{Mg}^{2+}:$ Magnésium ; $\quad *$ : augmentation significative $(\mathrm{p}<0,05)$.

Tableau 4: Effet de Aphro sur le cholestérol total et les triglycérides chez les rats traités et les rats témoins.

\begin{tabular}{clllll}
\hline \multicolumn{1}{c}{ Paramètres } & Lots & J0 & J14 & J28 \\
\hline \multirow{3}{*}{ Cholestérol T (g/L) } & Lot $1($ Témoin) & $0,90 \pm 0,10$ & $1,10 \pm 0,11$ & $1,11 \pm 0,14$ \\
& Lot 2 $(5,75 \mathrm{mg} / \mathrm{Kg})$ & $1,00 \pm 0,30$ & $1,10 \pm 0,12$ & $0,89 \pm 0,10$ \\
& Lot $3(11,5 \mathrm{mg} / \mathrm{Kg})$ & $1,02 \pm 0,40$ & $1,00 \pm 0,09$ & $0,96 \pm 0,13$ \\
& Lot $4(23 \mathrm{mg} / \mathrm{Kg})$ & $1,08 \pm 0,55$ & $1,10 \pm 0,09$ & $0,97 \pm 0,09$ \\
\hline \multirow{3}{*}{ Triglycériques $(\mathbf{g} / \mathbf{L})$} & Lot $1(\mathrm{Témoin})$ & $0,63 \pm 0,10$ & $0,60 \pm 0,09$ & $0,65 \pm 0,05$ \\
& Lot $2(5,75 \mathrm{mg} / \mathrm{Kg})$ & $0,59 \pm 0,09$ & $0,62 \pm 0,03$ & $0,61 \pm 0,07$ \\
& Lot $3(11,5 \mathrm{mg} / \mathrm{Kg})$ & $0,62 \pm 0,08$ & $0,59 \pm 0,09$ & $0,62 \pm 0,09$ \\
& Lot 4 $(23 \mathrm{mg} / \mathrm{Kg})$ & $0,63 \pm 0,10$ & $0,60 \pm 0,10$ & $0,62 \pm 0,04$ \\
\hline
\end{tabular}

Pas de différence significative ( $\mathrm{p}>0,05)$. 


\section{DISCUSSION}

Le suivi de l'évolution de la masse corporelle des rats traités avec Aphro n'a pas indiqué une perturbation sur le gain pondéral normal des rats traités aux doses de 11,5 et 23 $\mathrm{mg} / \mathrm{kg}$ de poids corporel par rapport aux rats du lot témoin. Cependant, un gain plus élevé mais non significatif a été observé chez les animaux du lot 2 traités à la dose de $5,75 \mathrm{mg} / \mathrm{kg}$ comparativement au lot témoin. Ces résultats suggèrent que le phytomédicament Aphro n'affecte pas négativement la prise du poids corporel chez les rats.

$\mathrm{Au}$ cours de la présente étude, le nombre de globules blancs, de globules rouges, des plaquettes sanguines et le taux d'hémoglobine des lots traités avec le phytomédicament n'ont indiqué aucune variation significative aux jours $\mathrm{J} 0, \mathrm{~J} 14$ et $\mathrm{J} 28$ comparativement au lot témoin. Ces résultats indiqueraient que le phytomédicament Aphro ne serait pas toxique chez les rats aux doses de $5,75 \mathrm{mg} / \mathrm{kg}, 11,5$ et $23 \mathrm{mg} / \mathrm{kg}$ de poids corporel durant 28 jours d'administration répétée. Ces résultats pourraient suggérer que l'utilisation de Aphro sur une durée de 28 jours n'a aucune incidence sur les cellules sanguines humaines. Gomé et al. (2011) ont observé des résultats similaires aux nôtres chez des rats traités avec l'extrait aqueux de Passiflora foetida (Passifloraceae) sur une période de 28 jours aux doses allant de 600 à $1200 \mathrm{mg} / \mathrm{kg}$ de poids corporel. Leurs résultats n'ont pas indiqué une perturbation des globules blancs et des thrombocytes chez les rats traités par rapport aux témoins.

En effet, selon Rhiouani et al. (2008), tout changement des paramètres hématologiques a une valeur prédictive pour l'intoxication humaine, lorsque les données sont traduites à partir des études réalisées sur des animaux. Nos résultats diffèrent de ceux de Gbogbo et al. (2018) qui ont montré que l'administration de 500 et $1000 \mathrm{mg} / \mathrm{kg}$ poids corporel du phytomédicament obtenu à partir l'extrait total aqueux de l'écorce de la tige de Spondias mombin L., entraîne une diminution significative du nombre de globules rouges, du taux d'hémoglobine et une augmentation des plaquettes sanguines chez les rats.
Les résultats ont également montré que l'administration du phytomédicament Aphro aux animaux n'a eu aucun effet sur la variation des marqueurs biochimiques hépatiques. Ces résultats signifient que Aphro n'a eu aucun effet délétère sur la fonction hépatique de ces derniers. En effet, l'aspartate aminotransférase, l'alanine aminotransférase, la phosphatase alcaline et à un degré moindre la gammaglutamyltranspeptidase sont des enzymes couramment analysées pour évaluer les dommages hépatiques (Dillon et Miller, 2016). Toute nécrose cellulaire, destruction du parenchyme hépatique ou une augmentation de la perméabilité membranaire des hépatocytes entraine l'écoulement de ces enzymes dans la circulation sanguine et donc l'augmentation de leurs taux sériques (Pariente, 2013 ; Manda et al., 2017). Leur activité est proportionnelle au degré de dommages hépatiques et sont donc bons indicateurs de l'hépatotoxicité (Pariente, 2013). Koné et al. (2009) ont également montré dans leurs travaux une absence de perturbation sur quelques marqueurs biochimiques hépatiques après administration d'un phytomédicament chez des rats Wistar albinos sur une période de 28 jours aux doses de 3,5; 35 et $350 \mathrm{mg} / \mathrm{kg}$ de poids corporel. Ces résultats diffèrent cependant de ceux obtenus par Gbogbo et al. (2014). Ces derniers ont montré que l'administration subaiguë de l'extrait total aqueux des écorces de tige de spondias mombin L. entrânerait une augmentation graduelle des marqueurs hépatiques au cours du traitement.

Les paramètres constitués de créatinine, acide urique, calcium et magnésium ont permis d'évaluer l'effet du phytomédicament Aphro sur la fonction rénale chez les rats.

Les résultats ont montré une augmentation significative $(\mathrm{p}<0,05)$ du taux de créatinine chez les rats traités avec le phytomédicament Aphro aux doses de 11,5 et $23 \mathrm{mg} / \mathrm{kg}$ de poids corporel par rapport aux témoins notamment à $\mathbf{J} 28$. Selon Ariarajah et al. (2011) la créatinine est le paramètre d'exploration rénale le plus utilisé et son augmentation sérique peut être expliquée par une atteinte de la fonction rénale. D'autres auteurs ont également montré l'effet toxique de 
certains phytomédicaments sur la fonction rénale par l'augmentation du taux sérique de la créatinine (Ashraf et al., 2013 ; Gbogbo et al., 2014 ; Awotundea et al., 2019). Hormis la créatinine, les autres paramètres n'ont pas connu de perturbations notables au cours de l'étude.

Le bilan lipidique effectué grâce au dosage du cholestérol total et des triglycérides n'a indiqué aucune différence statistiquement significative de J0 à J28 entre les lots des rats traités et celui des rats témoins. Des résultats similaires ont été obtenus dans d'autres travaux réalisés sur des phytomédicaments chez les rats Wistar (Koné et al., 2009 ; Adebayo et al., 2010). En somme, Aphro n'a pas provoqué de perturbation sur les paramètres lipidiques lorsqu'il a été administré aux doses de $5,75 \mathrm{mg} / \mathrm{Kg}, 11,5$ et $23 \mathrm{mg} / \mathrm{kg}$ de poids corporel sur une période de 28 jours.

\section{Conclusion}

L'étude de la toxicité par administration répétée sur une période de 28 jours et aux doses de 5,$75 ; 11,5$ et $23 \mathrm{mg} / \mathrm{kg}$ de poids corporel a permis de montrer chez les rats Wistar que le phytomédicament Aphro ne perturbe pas l'accroissement de la masse corporelle lors de son utilisation. En outre, Aphro n'a eu aucun effet néfaste sur les trois lignées cellulaires hématologiques ainsi que sur les paramètres biochimiques de la fonction hépatique et le bilan lipidique. Par contre, une augmentation significative de la créatinine sérique a été

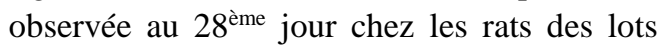
traités avec le phytomédicament Aphro aux doses de 11,5 et $23 \mathrm{mg} / \mathrm{kg}$ de poids corporel. Il ressort donc de cette étude qu'une administration prolongée de Aphro sur une période de 28 jours peut entraîner des risques de toxicité au niveau rénal. Ces résultats nous interpellent donc à la vigilance quant à l'utilisation à long terme des préparations médicamenteuses à base de plantes vendues sur nos marchés.

\section{CONFLIT D'INTERETS}

Les auteurs déclarent ne pas avoir de conflit d'intérêts.

\section{CONTRIBUTIONS DES AUTEURS}

MG, RYK et FTA ont réalisé l'étude expérimentale et ont soumis le projet d'article. MK, EKK et PAY ont formaté le manuscrit sous tous les aspects selon les instructions de la revue.

\section{REMERCIEMENTS}

Nous remercions toute l'équipe de l'animalerie du laboratoire de Physiologie, Pharmacologie et Pharmacopée de l'Université Nangui Abrogoua pour la mise à notre disposition des rats pour les expérimentations.

\section{REFERENCES}

Adebayo AH, Zeng GZ, Fan JT, Ji CJ, He WJ, $\mathrm{Xu}$ JJ, Zhang YM, Akindahunsi AA, Roseline Kela R, Tan NH. 2010. Biochemical, haematological and histopathological studies of extract of Ageratum conyzoides L. in Sprague Dawley rats. J. Med. Plants Res., 4(21): 2264-2272.

DOI: http://10.5897/JMPR10.470

Ariarajah N, Gerstel E, Martin PY, Ponte B. 2011. Biomarqueurs dans l'insuffisance rénale aiguë. Rev. Med. Suisse, 7 : 490494.

DOI: http://www.revmed.ch.2mars2011

Ashraf H, Heidari R, Nejati V, Ilkhanipoor M. 2013. Aqueous extract of Berberis integerrima root improves renal dysfunction in streptozotocin induced diabetic rats. Avicenna J. Phytomed., 3(1): $\quad 82-90 . \quad$ DOI: https://www.ncbi.nlm.nih.gov/pmc/articl es/PMC4075691

Awotundea OS, Adewoyeb SO, Dhanabal PS, Hawumbad J. 2019. Subacute toxicity study of aqueous root extract of Terminalia schimperiana in male Wistar rats. Toxicol. Rep., 6 : 825-832. DOI: https://doi.org/10.1016/j.toxrep.2019.07. 006

Bernard S. 1989. Biochimie Clinique Instruments et Techniques de Laboratoire- Diagnostiques Médicaux Chirurgicaux (2 $2^{\text {nd }}$ édn). Ed. Maloine: Paris; 153-156. 
Connerty HV, Briggs RA. 1966. Determination of serum calcium by means of orthocresolphthalein complexone. Am. J. Clin. Path., 45(3) : 200-296. DOI: 10.1093/ajcp/45.3.290

Dillon J, Miller MH. 2016. Gamma glutamyl transferase 'To be or not to be' a liver function test? Ann. Clin. Biochem., 53(6) : 629-631. DOI: 10.1177/0004563216659887

Dro B, Soro D, Koné MW, Bakayoko A, Kamanzi K. 2013. Evaluation de l'abondance de plantes médicinales utilisées en médecine traditionnelle dans le Nord de la Côte d'Ivoire. J. Anim. Plant Sci., 3(17): 2631-2646. DOI: http://www.m.elewa.org/JAPS

Farrell EC.1984. Magnesium In Clinical Chemistry, Kaplan LA (ed.). The C.V. Mosby Co.: St Louis Toronto. Princeton; 1065-1069.

Gbogbo M, Koné M, Bleyere NM, Yao KE, Yapo AP. 2014. Effect of total aqueous stem bark extract of Spondias mombin L. on some biochemical and anthropometric parameters in Wistar albino rats. Int. J. Biosci., $\quad 4(7)$ : $1-8 . \quad$ DOI: http://dx.doi.org/10.12692/ijb/4.7.1-8

Gbogbo M, Touré A, Kouadio YE, Oussou NJB, Koné M, Diby YB, Yapo AP. 2018. Toxicity Assessment of an Aqueous Extract of the Stem Bark of Spondias mombin (Anacardiaceae) in Wistar albino rats. Int. J. Curr Microbiol. Appl. Sci., 7(1): 3625-3635. DOI: https://doi.org/10.20546/ijcmas.2018.702 .426 .

Gomé BM, Kouakou K, Touré A, Traoré F. 2011. Étude de la toxicité aiguë et subchronique de l'extrait aqueux de Passiflora foetida Linn. (Passifloraceae) chez les rats et les souris. Int. J. Biol. Chem. Sci., 5(5): 1777-1789. DOI : http://dx.doi.org/10.4314/ijbcs.v5i5.1

Irie-n'guessan GA, Effo EK, Koua DBK, Kouakou LS, Djadji LTA, Adepo AA, Diarrassouba N, Kouakou-Siransy GN. 2019. La toxicité subaiguë de l'écorce de racines de Dichrostachys cinerea (L.) Wight et Arn. (Fabaceae). Int. J. Biol.
Chem. Sci., 13(2): 836-848. DOI: https://dx.doi.org/10.4314/ijbcs.v13i2.21

Kandé B, Yao K, Allah KE, Koné MW. 2018. Enquête sur l'utilisation et l'effet des médicaments à base de plantes chez les patients hépatiques hospitalisés du Centre Hospitalier Universitaire de Cocody en Côte d'Ivoire. J. Appl. Biosci.; 130: 13220 - 13231 .

DOI: https://10.4314/jab.v130i1.9

Kaplan A. 1984. Triglycerides In: Clinical Chemistry (ed. 437 and Lipids). The C.V. Mosby Co. St Louis: Torronto. Princeton; 1194-1206.

Koné M, Bléyere NM, Yapo AP, Vangah MO, Ehilé EE. 2009. Evaluation de la toxicité d'un extrait aqueux de Sacoglottis gabonensis (Baille) Urban (Humiriaceae) chez les rongeurs, une plante utilisée dans le traitement de l'ulcère de Buruli en Côte d'Ivoire. Int. J. Biol. Chem. Sc., 3(6): 1286-1296.

DOI : https://10.4314/ijbcs.v3i6.53147

Kroa E, Diaby B, Niaré A, Traoré Y, Ahoussou EM, Yao GHA, Coulibaly GS, Kouassi D. 2014. Analyse de la collaboration entre médecines traditionnelle et moderne dans la région du Sud Bandama (Côte d'Ivoire). Revue CAMES-Série Pharm. Méd. Trad. Afr., 17(1):21-27. DOI : http://publication.lecames.org/index.php/ pharm/article/view/237/137

Manda P, Manda O, Obouo M, Manda V, Kroa E, Dano SD. 2017. Étude des toxicités aiguë et subaiguë du remède nature utilisé dans le traitement du paludisme. Rev. Ivoir. Sci. Technol., 29 : 145 - 158. http://www.revist.ci

Murray RL. 1984. Aspartate aminotransferase. In Clinical Chemistry, Kaplan A, Peace AL (Eds). The C.V. Mosby Co. : St Louis, Toronto, Princeton ; 1112- 1116.

Naito HK. 1984. Cholesterol. In Clinical Chemistry, Kaplan A (Ed). The C. V. Mosby Co.: St Louis Toronto Princeton; 1194-11206.

Najem M, Belaidi R, Harouak H, Bouiamrine EH, Ibijbijen J, Nassiri L. 2018. Occurrence de plantes toxiques en phytothérapie traditionnelle dans la 
région du Moyen Atlas central Maroc. $J$. Anim. Plant Sci., 35 (2) : 5651-5673. DOI: http://www.m.elewa.org/JAPS

OCDE. 1998. Série sur les principes de bonnes pratiques de laboratoire et vérification du respect de ces principes. ENV/MC/CHEM; 17 : 22-23.

OMS. 2002. Stratégie de l'OMS pour la médicine traditionnelle pour 2002- 2005. Organisation Mondiale de la Santé, Genève.

OMS. 2004. Qualité et innocuité des médicaments : systèmes de réglementation. Cinquante-septième Assemblée mondiale de la santé. Point 12.12 de l'ordre du jour provisoire du 19 avril 2004, Genève.

Owens C, Baergen R, Puckettc D. 2014. Online Sources of Herbal Product Information. Amer. J. Med., 127(2): 109-115. DOI: http://10.1016/j.amjmed.2013.09.016

Pariente A. 2013. Cytolyse hépatique (augmentation des aminotransferases) chez l'adulte. Hepato. Gastro., 20 : 630639. DOI: http://10.1684/hpg.2013.0916
Rhiouani H, El-Hilaly J, Israili ZH, Lyoussi B. 2008. Acute and sub-chronic toxicity of an aqueous extract of the leaves of Herniaria glabra in rodents. $J$. Ethnopharmacol., 118(3): 378-386. DOI: http://10.1016/j.jep.2008.05.009

Rosalki SB, Foo AY, Burlina A, Prellwitz W, Stieber P, Neumeier D, Klein G, Poppe WA, Bodenmüller H. 1993. Multicenter evaluation of Iso-ALP test kit for measurement of bone alkaline phosphatase activity in serum and plasma. Clin Chem., 39(4): 648-652. DOI : http://intl.clinchem.org/cgi/content/abstr act $/ 39 / 4 / 648$

Stickel F, Patsenker E, Schuppan D. 2005. Herbal hepatotoxicity. J. Hepatol., 43: 901-910. DOI: http://10.1016/j.jhep.2005.08.002

Tietz NW, Burtis CA, Ashwood ER, Saunders WB. 1999. Textbook of Clinical Chemistry (3rd Edn); 676-684.

Wenger C. 1984. Alkaline phosphatase. In Clinical Chemistry, Kaplan LA (Ed.). The C.V. Mosby Co. Si Louis: Tronto Princeton ; 1094-1098. 